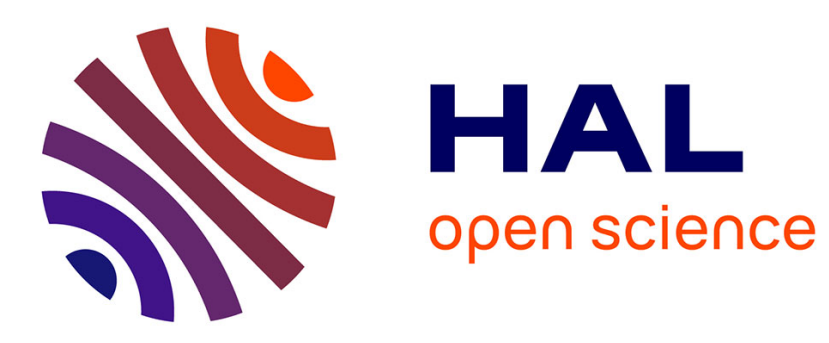

\title{
Large-Scale Production of Algal Biomass: Photobioreactors
}

\author{
Jeremy Pruvost, Jean-François Cornet, Laurent Pilon
}

\section{To cite this version:}

Jeremy Pruvost, Jean-François Cornet, Laurent Pilon. Large-Scale Production of Algal Biomass: Photobioreactors. Faizal Bux; Yusuf Chisti. Algae Biotechnology: Products and Processes, Springer, pp.41-66, 2016, Green Energy and Technology, 10.1007/978-3-319-12334-9_3 . hal-02539922

\section{HAL Id: hal-02539922 \\ https://hal.science/hal-02539922}

Submitted on 10 Apr 2020

HAL is a multi-disciplinary open access archive for the deposit and dissemination of scientific research documents, whether they are published or not. The documents may come from teaching and research institutions in France or abroad, or from public or private research centers.
L'archive ouverte pluridisciplinaire HAL, est destinée au dépôt et à la diffusion de documents scientifiques de niveau recherche, publiés ou non, émanant des établissements d'enseignement et de recherche français ou étrangers, des laboratoires publics ou privés. 


\title{
Large-Scale Production of Algal Biomass: Photobioreactors
}

\author{
Jeremy Pruvost, Jean-François Cornet and Laurent Pilon
}

\begin{abstract}
Photobioreactors have been used extensively for the cultivation of microalgae for a variety of applications from biofuels to high value products. The ability to cultivate monocultures of algae with high biomass yields and significantly smaller footprints has made photobioreactors a very attractive technology for specific applications. This chapter deals with photobioreactor design, application, efficiencies, and factors affecting their performance.
\end{abstract}

Keywords Microalgae - Photobioreactors - PBR productivity - Photoinhibition • Biomass production

\section{Nomenclature}

$A \quad$ Local specific radiant energy absorbed $\left(\mu \mathrm{mol} \mathrm{s}{ }^{-1} \mathrm{~kg}^{-1}\right)$

$a_{\text {light }}$ Specific illuminated area for the photobioreactor $\left(\mathrm{m}^{-1}\right)$

$C_{\mathrm{X}} \quad$ Biomass concentration $\left(\mathrm{kg} \mathrm{m}^{-3}\right)$

$D \quad$ Dilution rate $\left(\mathrm{h}^{-1}\right.$ or $\left.\mathrm{s}^{-1}\right)$

$E_{a} \quad$ Mass absorption coefficient $\left(\mathrm{m}^{2} \mathrm{~kg}^{-1}\right)$

$f_{d} \quad$ Design dark volume fraction of any photobioreactor (dimensionless)

$G \quad$ Local irradiance $\left(\mu \mathrm{mol} \mathrm{s}{ }^{-1} \mathrm{~m}^{-2}\right)$

$G_{\mathrm{c}} \quad$ Compensation irradiance value $\left(\mu \mathrm{mol} \mathrm{s}{ }^{-1} \mathrm{~m}^{-2}\right)$

$K \quad$ Half saturation constant for photosynthesis $\left(\mu \mathrm{mol} \mathrm{s} \mathrm{s}^{-1}\right.$ )

\author{
J. Pruvost $(\bowtie)$ \\ GEPEA, Université de Nantes, CNRS, UMR 6144, BP 40637 bd de l'Université, CRTT, \\ 44602 Saint-Nazaire Cedex, France \\ e-mail: jeremy.pruvost@univ-nantes.fr \\ J.-F. Cornet \\ ENSCCF, Institut Pascal-UMR-CNRS 6602, Clermont Université, BP 10448, 63000 \\ Clermont-Ferrand, France \\ L. Pilon \\ Henry Samueli School of Engineering and Applied Science, University of California, \\ Los Angeles, 420 Westwood Plaza, Los Angeles, CA 90095, USA
}


$L \quad$ Depth of the rectangular photobioreactor (m)

$M_{X} \quad$ C-molar mass for the biomass $\left(\mathrm{kg}_{\mathrm{X}} \mathrm{mol}_{\mathrm{X}}^{-1}\right)$

$q \quad$ Photon flux density on a given surface (PFD) $\left(\mu \mathrm{mol} \mathrm{s}{ }^{-1} \mathrm{~m}^{-2}\right)$

$Q \quad$ Volume liquid flow rate $\left(\mathrm{m}^{3} \mathrm{~d}^{-1}\right)$

$r_{\mathrm{X}} \quad$ Biomass volumetric growth rate (productivity) $\left(\mathrm{kg} \mathrm{m}^{-3} \mathrm{~s}^{-1}\right.$ or $\mathrm{kg} \mathrm{m}^{-3} \mathrm{~h}^{-1}$ )

$S_{\text {light }}$ Illuminated surface of the photobioreactor $\left(\mathrm{m}^{2}\right)$

$\mathrm{P}_{\mathrm{S}} \quad$ Areal biomass productivity $\left(\mathrm{kg} \mathrm{m}^{-2} \mathrm{~d}^{-1}\right)$

$P_{\mathrm{V}} \quad$ Volumetric biomass productivity $\left(\mathrm{kg} \mathrm{m}^{-3} \mathrm{~d}^{-1}\right)$

$t \quad$ Time (days or s)

$V_{\mathrm{r}} \quad$ Photobioreactor volume $\left(\mathrm{m}^{3}\right)$

$x_{d} \quad$ Diffuse fraction for incident PFD at any location (-)

$z \quad$ Depth of culture (m)

\title{
Greek Letters
}

$\alpha \quad$ Linear scattering modulus (dimensionless)

$\beta \quad$ Inclination of the photobioreactor surface (rad)

$\gamma \quad$ Fraction for working illuminated volume in the photobioreactor (dimensionless)

$\delta \quad$ Extinction coefficient for the two-flux method $\left(\mathrm{m}^{-1}\right)$

$\theta$ Incident angle (defined from the outward normal of the PBR) (rad)

$\lambda \quad$ Wavelength (m)

$\rho_{\mathrm{M}}$ Maximum energy yield for photon conversion (dimensionless)

$\tau_{p} \quad$ Residence time (h)

$\tau_{\lambda} \quad$ Absorption optical thickness, $\tau_{\lambda}=\mathrm{Ea}_{\lambda} C_{\mathrm{X}} / a_{\text {light }}$ (dimensionless)

$\varphi_{X}^{\prime} \quad$ Biomass mole quantum yield for the Z-scheme of photosynthesis $\left(\mathrm{mol}_{\mathrm{X}} \mu \mathrm{mol}_{\mathrm{hv}}^{-1}\right)$

\section{Subscripts}

max Related to maximum available value

opt Related to the optimal value for residence time

\section{Other}

$\langle X\rangle=\frac{1}{V} \iiint_{V} X \mathrm{~d} V \quad$ Spatially-averaged property

$\bar{X}=\frac{1}{\Delta t} \int_{\Delta t} X \mathrm{~d} t \quad$ Time-averaged property

\author{
Abbreviations \\ PAR Photosynthetically active radiation \\ PBR Photobioreactor \\ PFD Photon flux density
}




\section{Cultivation Systems}

\subsection{Requirements for Photosynthetic Growth and Possible Limitations}

Photosynthetic growth in standard autotrophic conditions is based on the assimilation, under illumination, of inorganic carbon and mineral nutrients dissolved in the medium. Cultivation of photosynthetic microorganisms requires the following: (1) sunlight or an artificial light source, with an appropriate emission spectrum in the photosynthetically active radiation (PAR) region, ranging between 400 and $700 \mathrm{~nm}$, (2) an inorganic carbon source, such as dissolved $\mathrm{CO}_{2}$, (3) mineral nutrients including major nutrients such as $\mathrm{N}, \mathrm{S}, \mathrm{P}$ and micronutrients such as $\mathrm{Mg}$, $\mathrm{Ca}, \mathrm{Mn}, \mathrm{Cu}, \mathrm{Fe}$, etc., and (4) a favorable set of culture conditions including $\mathrm{pH}$, temperature, and dissolved oxygen.

Quantitatively, the main variables affecting photosynthetic growth and productivity of microalgae cultivation systems are (1) the incident light characterized by its photons flux density (PFD) with given angular distribution and spectrum, (2) the concentrations of various compounds in the liquid phase affecting growth such as dissolved inorganic carbon, dissolved oxygen, and growth mineral nutrients, and (3) culture conditions such as $\mathrm{pH}$, temperature, and possible biological contamination. Designing and operating a microalgal cultivation system aims to optimize these conditions with the objective of maximizing growth. Control of these growth conditions is significantly more complex in industrial scale systems under outdoor conditions than in indoor benchtop systems. In fact, maintaining optimal growth conditions is very difficult to achieve for microalgae cultivation systems under highly variable outdoor conditions and scaling up the bioprocess features' major technical challenges discussed in this chapter.

\subsection{Open Systems and Closed Photobioreactors}

Open systems, such as natural ponds and raceways, are currently the most employed technology for outdoor solar cultivation. In fact, they have been used for several decades at industrial scale (Borowitzka 1999; Carvalho et al. 2006; Molina Grima et al. 1999; Morweiser et al. 2010; Pruvost 2011; Pulz 2001; Richmond 2004a; Ugwu et al. 2008). The main limitations of open systems are inherent to their operating principles. First, they are exposed to high risks of biological contamination by other microalgae species, bacteria, and/or predators owing to the direct contact of the culture with the atmosphere. Therefore, only resistant species can be cultivated for long periods of time. The large interface between the culture and the atmosphere also renders the control of culture conditions difficult. For example, it is difficult to maintain an optimal temperature although open systems are less subject to overheating than closed systems. In addition, the relatively low atmospheric $\mathrm{CO}_{2}$ concentration generally results in small concentrations of dissolved carbon in the 
culture medium, often insufficient to meet the needs of photosynthetic microorganisms in intensive biomass production. Thus, a carbon source can be added by injecting $\mathrm{CO}_{2}$ gas (or chemical carbonate). However, a significant part of dissolved inorganic carbon is inevitably degassed into the atmosphere. In practice, this makes carbon limitation difficult to prevent entirely in open systems.

Closed systems, often called "photobioreactors" (PBRs), reduce risks of external contamination and provide better control of growth conditions. For example, $\mathrm{CO}_{2}$ can be sparged into the PBRs. Then, the larger gas partial pressure in the bubbles and PBR headspace prevent carbon limitation. However, PBRs also suffer from several limitations inherent to their operating principles. First, culture confinement increases the risk of biofilm formation on the PBR walls. It leads to oxygen accumulation in the culture which can have possible toxic effects on photosynthetic growth. It may also cause overheating of the culture especially under solar radiation due to the large amount of infrared radiation absorbed by the culture medium (Borowitzka 1999; Carvalho et al. 2011; Grobbelaar 2008; Torzillo et al. 1996). Unlike challenges faced by open systems, those affecting closed PBRs can be overcome in part by appropriate engineering solutions such as optimizing mixing conditions to increase heat transfer and gas-liquid mass transfer or to prevent biofilm formation. Once all these challenges have been addressed, light remains the only limiting factor. In other words, the amount of light received and its use by the culture will determine the productivity of the system.

Finally, solutions designed to overcome the different technical challenges previously mentioned result, most often, in increased cost and complexity. Current industrial scale biomass production is mainly performed in large open systems because they are easier to build and operate than PBRs. However, PBR technology offers higher potential for improvement in terms of productivity and efficiency. Great efforts are currently underway to develop new technologies devoted to industrial-scale production in PBRs.

\subsection{Photobioreactors Principles}

There exists a wide variety of PBRs technologies such as tubular, cylindrical, or flat panel systems, as illustrated in Fig. 1. This diversity of PBR designs results from various attempts to optimize light capture while satisfying other practical constraints related to (i) engineering design including system integration, scale of production, materials selection, and cost and to (ii) system operation concerned with $\mathrm{CO}_{2}$ bubbling, oxygen removals, temperature and $\mathrm{pH}$ regulation, nutrient delivery, etc. Numerous reports and publications can be found in the literature on the various PBR technologies available. All of them have advantages and limitations in terms of control of culture conditions, culture confinement, hydrodynamics conditions, easiness to scale up, construction cost, biomass productivity, and energy efficiency (Borowitzka 1999; Carvalho et al. 2006; Grima et al. 1999; Morweiser et al. 2010; Pruvost 2011; Pulz 2001; Ugwu et al. 2008). Regardless of the PBR 
(a)

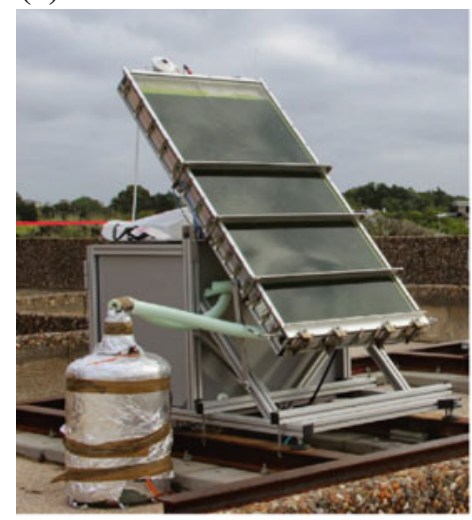

(c)

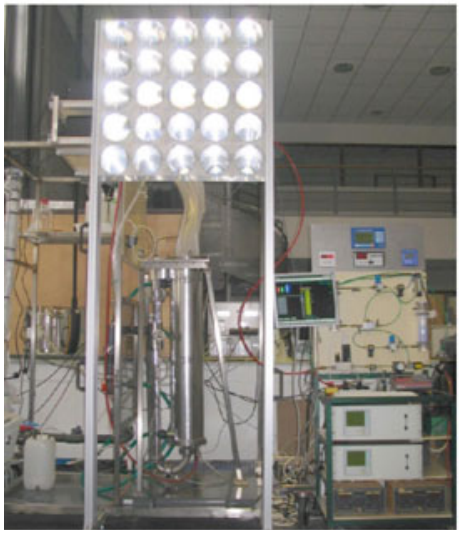

(b)

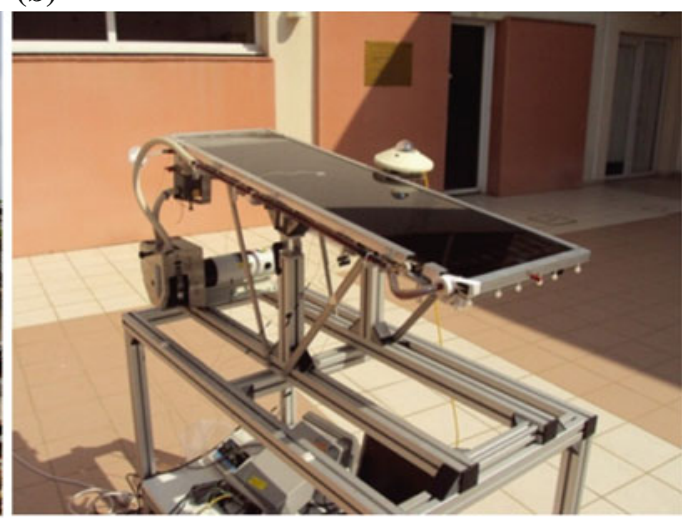

Fig. 1 Examples of solar photobioreactor technologies. a Flat panel solar PBR (GEPEA, University of Nantes, France). b AlgoFilm(C) solar PBR (ultrathin PBR) (GEPEA, University of Nantes, France). c DiCoFluV(C solar photobioreactor with Fresnel lenses for sun capture and lateral diffusing optical fibers inside the reactor (Institut Pascal, Clermont-Fd, France)

concept, the goal is to provide sufficient control of the culture conditions to make the process only limited by the amount of light supplied and the photosynthetic process in the culture. The photon flux densities (PFDs) incident onto the PBR surface and locally available inside the culture are major parameters. Although maximizing light intercepted must be an obvious consideration of any microalgal cultivation system (as it is for any solar process), other constraints also have to be considered. For example, using the airlift method for mixing will preclude horizontal geometries. In addition, shading must be accounted for when arranging vertical or tilted systems on a given land area. Therefore, optimizing photobiological cultivation systems proves more complex than other traditional solar-driven processes, such as photovoltaic panels, where the amount of intercepted light is the only operating parameter of any given panel technology. 


\subsection{Surface and Volumetric Illuminations}

Light can be supplied in two general ways, either by direct illumination of the cultivation system or by distributing light sources inside the culture volume. Then, one distinguishes between surface-illuminated and volumetrically illuminated systems, respectively. Most cultivation systems fall in the simpler surface-illuminated category (Carvalho et al. 2006; Morweiser et al. 2010; Richmond 2004a; Ugwu et al. 2008). As for any solar processes, various positioning options have been considered including systems positioned horizontally (Acién Fernández et al. 2001; Oswald 1988; Molina et al. 2001), vertically (Chini Zitelli et al. 2000, 2006; Pulz 2001), and in few cases, tilted (Doucha and Livansky 2006; Lee and Low 1991; Richmond and Cheng-Wu 2001). However, maximizing the incident solar radiation flux is not trivial. It obviously depends on the longitude and latitude of the system's location and on the day of the year. For example, horizontal systems are best suited for locations close to the Equator (latitude $0^{\circ}$ ). For higher latitudes, it is necessary to tilt the system exposed surface to maximize the amount of light collected. Roughly speaking, the optimum inclination angle with respect to Earth surface which maximizes light capture over the year on a fixed PBR corresponds to the latitude of the PBR location (Duffie and Beckman 2006; Hu et al. 1996; Pruvost et al. 2012; Richmond and Cheng-Wu 2001). Inclination angle can also be adjusted as a function of time to optimize light capture. For example, flat panel equipped with sun-tracking systems were tested by Hindersin et al. (2013). This method not only maximized light capture during the day but also prevented excessive incident irradiation on the systems around noon, by temporarily setting the illuminated surface of the PBR perpendicular to the sun collimated irradiation.

Volumetrically illuminated systems require more complex technologies than surface-illuminated systems. However, they enable the optimization of the light delivery and use in the culture. First, inserting light sources in the volume of the culture guarantees maximal use of the collected or emitted photons. Second, and more interestingly, internal lighting allows light to be "diluted." Increasing PFD leads to higher volumetric productivity but associated with a progressive decrease in the conversion yield, due to photosynthesis saturation. By diluting the light incident on the system's surface into the volume of the culture, a larger yield can be maintained. This is of particular interest in outdoor PBRs exposed to sunlight. In this case, solar radiation incident on a given surface is collected using, for example, a parabolic solar collector. It is then delivered to the culture in a controlled manner, using optical fibers (Cornet 2010; Csogör et al. 2001) or light guides (Pilon et al. 2011), for example. Because of the large PFD characteristic of solar conditions, an increase in surface productivity can be achieved. Note that the optical connection between the light collection device and the light delivery system needs to be carefully designed as it can be the source of major optical losses. Furthermore, light dilution can be combined with a solar tracking system, giving an additional possibility of optimization by maximizing light intercepted as the sun travels in the sky (Hindersin et al. 2013). A full description of such a principle has been described by 
Cornet (2010) with a volumetrically lightened photobioreactor based on the "DiCoFluV" concept. Despite their promise, only a few examples of volumetrically illuminated PBRs can be found in the literature (Cornet 2010; Csogör et al. 2001; Hsieh and Wu 2009; Ogbonna et al. 1996; Zijffers et al. 2008). This is mainly explained by the increase in technological complexity and by the difficulty in scaling up PBR systems to large surface areas.

\section{Photobioreactor Engineering and Scaling Rules}

\subsection{Maximizing Biomass Production}

Most growth limitations previously mentioned can be avoided, or at least greatly reduced, thanks to proper engineering design of the cultivation system. This is especially true for nutrient and $\mathrm{CO}_{2}$ limitations in closed PBRs. Note, however, that light limitation cannot be avoided due to the rapid light attenuation in the culture and to the large energy requirement of photosynthesis. This simple yet important observation is central to the optimization of microalgae cultivation systems. One major practical consequence involves the need to develop PBR with geometries able to optimize light supply to the culture. But, as detailed hereafter, working under light limitation will also facilitate the design and control of efficient processes. Light will be indeed the only parameter to control. This implies however that the effects of light on the process should be accurately taken into account.

\subsection{Growth Limitations by Nutrient and Inorganic Carbon Sources}

In order to prevent mineral limitation, the growth medium must contain all the necessary macro- and micronutrients in sufficient quantities based on the expected biomass concentration. Stoichiometric equations can be used for this purpose, or nutrient concentrations can be monitored and adjusted during cultivation. The interested reader is referred to studies in which this method has been applied to various species (Pruvost et al. 2009; Pruvost 2011).

Inorganic carbon source comes from $\mathrm{CO}_{2}$ gas dissolved in the culture medium or from directly adding chemical carbonate compounds in the medium. In both cases,

a minimum amount of total dissolved carbon (TDC) of about $5-10 \mathrm{mM}$ is necessary to avoid carbon limitation on the microorganisms' growth. When using gaseous $\mathrm{CO}_{2}$, the minimum TDC depends on the $\mathrm{pH}$, the biological consumption rate, and the gas-liquid mass transfer rate. The latter is affected not only by the magnitude of the mass transfer coefficient $k_{\mathrm{L}} a$ but also by the driving force of the carbon dissolution in water determined by thermodynamics equilibria of the chemical reactions involved. 
TDC can be determined by performing a mass balance analysis on the cultivation process or by directly monitoring TDC using a total inorganic carbon measurement, for example (Degrenne et al. 2010). This is analogous to oxygen dissolution in aerobic cultivation of yeasts or bacteria. The main significant difference lies in the fact that $\mathrm{CO}_{2}$ dissolution also affects the $\mathrm{pH}$ of the growth medium, i.e., excess supply of $\mathrm{CO}_{2}$ leads to acidification of the medium. In turn, this influences the amount and type of dissolved carbon $\left(\mathrm{CO}_{2}, \mathrm{HCO}_{3}{ }^{-}\right.$, and $\left.\mathrm{CO}_{3}{ }^{2-}\right)$ in the culture. Overall, the carbon feeding strategy requires maintaining the $\mathrm{pH}$ optimal for growth while averting carbon limitation. This may not be trivial considering that mineral nutrient consumption during growth can also participate in the water chemistry of carbon. However, in most cases, simple $\mathrm{CO}_{2}$ bubbling is usually sufficient for regulating both $\mathrm{pH}$ and TDC. This could be more difficult when ammonium is used as a nitrogen source since its consumption during photosynthetic growth also leads to acidification (Ifrim et al. 2014).

\subsection{The Light-Limited Regime}

The control of cultivation conditions such as $\mathrm{pH}$ and temperature can be challenging in practice, especially in outdoor conditions (Borowitzka 1999; Grobbelaar 2008; Richmond 2004a; Torzillo et al. 1996). These challenges, however, can be overcome with an adequate engineering and control of the cultivation system. If all cultivation conditions are kept at their optimal value, and nutrients are provided in adequate amounts, light-limited conditions should eventually occur. The light-limited regime has several major features. The first consequence is that, by definition, the culture is not subject to any other limitation. Thus, maximum biomass productivity can be achieved and is determined by the amount of light provided and its use by the culture (Takache et al. 2010; Pruvost 2011; Pruvost et al. 2011b, 2012; Pruvost and Cornet 2012). Any limitation other than light limitation would result in further decrease of biomass productivity while maximizing the PFD received onto the cultivation system increases the productivity. Note that this remains valid in the case of high PFD leading to photoinhibition of photosynthetic apparatus (PFD roughly superior to $400 \mu$ mole $_{\mathrm{hv}} \mathrm{m}^{-2} \mathrm{~s}^{-1}$ ). Special attention should be paid to light attenuation conditions to avoid or at least greatly reduce photoinhibition phenomena by operating the PBR to achieve complete light extinction in the culture, as described in detail in the next section. A second important consequence is that, in the light-limited regime, controlling the incident light and its effect on the process leads to the control of the entire cultivation system performance. This corresponds to the so-called "physical limitation» in chemical engineering, when the process is limited by one parameter whose control enables the control of the entire process. This last feature is essential to the efficient design and operation of photobiological cultivation systems. 


\subsection{Role of Light Attenuation Conditions and Absorption Rate}

The light-limited regime is a necessary but not sufficient condition to obtain maximal biomass productivity in any given PBR. Appropriate light transfer (or light attenuation) conditions have to be established inside the culture volume (Cornet and Dussap 2009; Pruvost 2011; Takache et al. 2010). On the one hand, if the biomass concentration is too low, part of the incoming photons is not absorbed and instead is transmitted through the culture (Fig. 2b, case A). This results in a loss of biomass productivity. In addition, the light received per cell is large and may lead to further decrease in productivity due to the increased photosynthetic dissipation. It may also induce a decrease in algal pigment content and thus lead to further increase in light transmission. As a consequence, the system becomes highly unstable resulting usually in culture washout. Thus, such conditions should be avoided in microalgae cultivation, especially for large incident PFD, typically larger than $200 \mu \mathrm{mole}_{\mathrm{hv}} \mathrm{m}^{-2} \mathrm{~s}^{-1}$. On the other hand, if the biomass concentration is too large, a dark zone appears inside the culture (Fig. 2b, case B). This dark zone is the direct consequence of light extinction by cells suspension, whose effect can be positive in the case of high illumination conditions by reducing photoinhibition effect and then increasing process stability (Carvalho et al. 2011; Grima et al. 1999; Richmond 2004b). Note that for microorganisms with respiration activity under illumination such as eukaryotic microalgae, a dark zone in the culture volume promotes respiration resulting in a loss of biomass productivity. Therefore, achieving the maximum biomass productivity requires in this case the exact condition of complete absorption of the incident light (Takache et al.2010), but without a dark zone in the culture volume, as illustrated in Fig. $2 \mathrm{~b}$, case $\mathrm{C}$. This condition is often referred to as luminostat mode. Note that it should not be confused with turbidostat mode referring to a turbidity-based regulation of a continuous culture. This condition has also been introduced as the " $\gamma=1$ " condition where $\gamma$ denotes the ratio of the volume of the PBR illuminated to the total volume of the culture (Cornet et al. 1994; Takache et al. 2010). For microorganisms with negligible respiration activity under illumination, such as prokaryotic cyanobacteria cells, fulfilling the condition of complete light absorption $(\gamma \leq 1)$ will be sufficient to reach the maximum biomass productivity.

Another way to represent the strong correlation between light attenuation conditions and the associated biomass productivity is to calculate the rate of photons absorption per unit volume of culture denoted by $\langle A\rangle$. The latter can be obtained by considering the spectral specific absorption coefficient $\mathrm{Ea}_{\lambda}\left(\mathrm{in} \mathrm{m}^{2} / \mathrm{kg}\right)$ of the cultivated species and the local spectral irradiance $\mathrm{G}_{\lambda}$ inside the culture of total volume $V_{R}$ (Aiba 1982; Cassano et al. 1995; Kandilian et al. 2013):

$$
\langle A\rangle=\frac{1}{V_{R}} \int_{\Delta \lambda} \iiint_{\mathrm{V}_{R}} \mathrm{Ea}_{\lambda} G_{\lambda} \mathrm{d} V \mathrm{~d} \lambda
$$

where $\Delta \lambda$ denotes the PAR range (400-700 nm). 
(a)

(Flowrate Q)

Photobioreactor

(Volume $V_{R}$ )

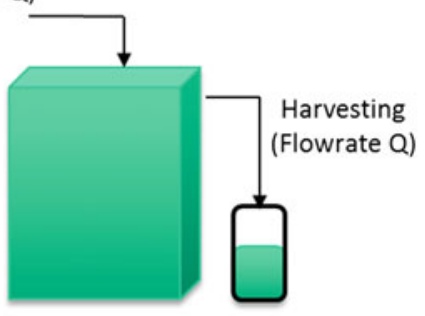

Residence time $\tau_{\mathrm{p}}$ and dilution rate $\mathrm{D} \quad \tau_{\mathrm{p}}=\frac{\mathrm{V}_{\mathrm{R}}}{\mathrm{Q}}\left(=\frac{1}{\mathrm{D}}\right)$

(b)

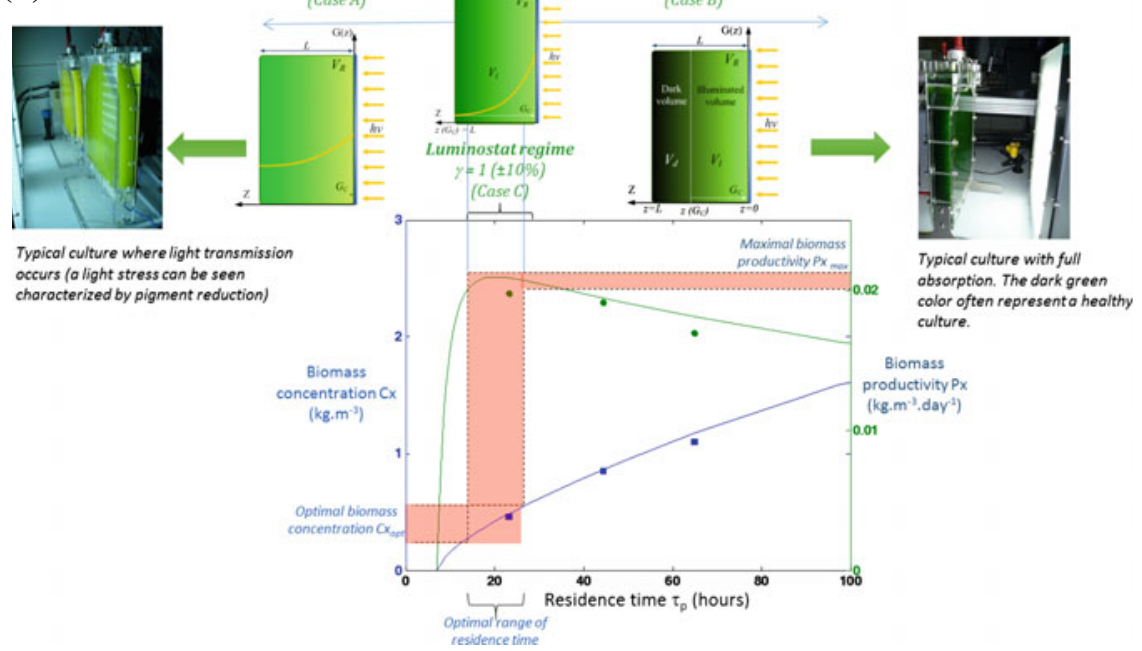

Fig. 2 Role of light attenuation conditions on biomass productivity in PBR. The illustration is here given for a PBR operated in continuous mode (a). The residence time (or dilution rate) applied allows controlling biomass concentration in the culture volume and then light absorption conditions, thus affecting the resulting biomass productivity. The relation with illuminated and dark zone repartition in the culture volume is given in (b), and the relation to the rate of photons absorption is given in (c). Both can be used independently to maximize biomass productivity of any cultivation system. Values are here given for Chlorella vulgaris (PFD $=200 \mu \mathrm{mole} \mathrm{m}^{2} \mathrm{~s}, \mathrm{PBR}$ depth $=0.04 \mathrm{~m})$

Increasing the biomass concentration in the cultivation system will result in a decrease of the rate of photons absorption by the cultivation system (Fig. 2c) due to stronger light attenuation and the resulting smaller irradiance $G_{\lambda}$. As a result, the maximum biomass productivity will be obtained for an optimal value of the rate of photons absorption, typically around $10-12 \mu$ mole $_{\mathrm{hv}} \mathrm{g}^{-2} \mathrm{~s}^{-1}$ (Fig. 2c). Note that this representation is consistent with the condition of luminostat regime $(\gamma=1)$, and 

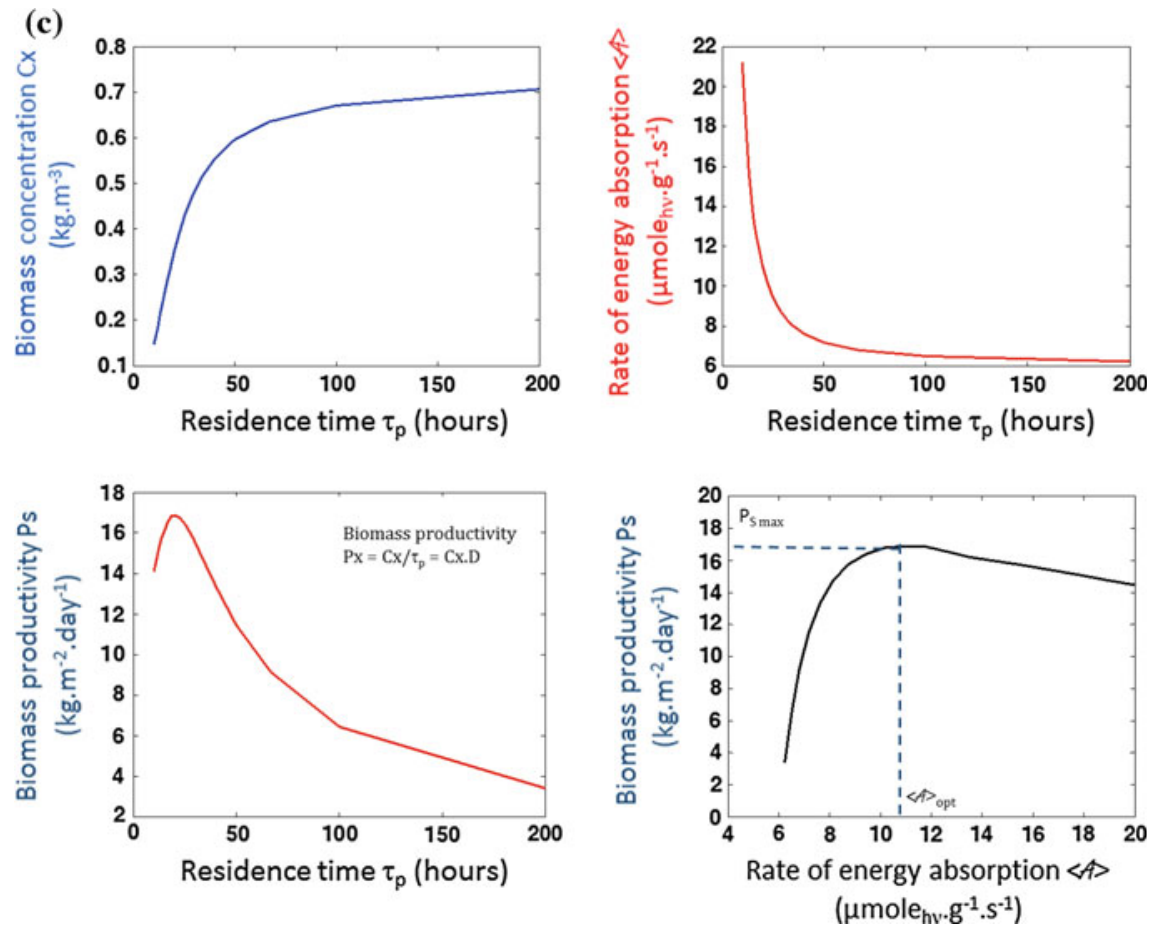

Fig. 2 (continued)

both approaches can be used to maximize the biomass productivity of any cultivation system.

One major consequence of the previous considerations is that, for any PBR and photosynthetic microorganism, light transfer from the collection site to the delivery inside the volume of the culture has to be taken into account. This analysis depends on the angular distribution of PFD, the PBR design, the biomass concentration, and the microorganisms' radiation characteristics. In practice, light attenuation conditions can be controlled by adjusting the biomass concentration. This can be achieved in continuous or semicontinuous cultivation mode, by modifying the residence time value $\tau_{\mathrm{p}}$ of the microorganisms in the cultivation system represented by the dilution rate $D$ such that $\tau_{\mathrm{p}}=1 / D=V_{\mathrm{r}} / Q$ where $Q$ represents the medium flow rate through the PBR. The reader is referred to Takache et al. (2010) for a detailed example of such optimization in continuous mode. In batch mode, $\langle A\rangle$ depends on time due to the continuous increase in biomass concentration making difficult the control and optimization of light absorption. To that effect, a simple and robust control strategy of the incident light has been recently proposed and demonstrated with benchtop PBRs (Kandilian et al. 2014).

In the specific case of solar production, maintaining optimal light attenuation conditions, even in continuous or semicontinuous operations, is far from a trivial 
task (Grognard et al. 2014; Hindersin et al. 2013; Pruvost et al. 2011a). The process is indeed strongly time dependent and driven by an uncontrolled and highly variable input, namely the solar incident flux. The growth kinetics of biomass is slow compared with the rapid variations in incident sunlight intensity and prevents the operator from establishing a luminostat regime. At best, a compromise needs to be found to determine the conditions approaching this ideal set point for most of the day. Whatever the case, light attenuation within the culture is not easy to determine. To do so, light transfer modeling is essential (Cornet et al. 1992a, b, 1995; Lee et al. 2014; Pilon et al. 2011; Pruvost and Cornet 2012; Pruvost et al. 2012). Furthermore, it can be associated with kinetics models of photosynthetic growth for a complete representation of the cultivation system characterized by its biomass concentration and biomass productivity. The reader is referred to the following studies for further information (Cornet 2010; Cornet and Dussap 2009; Lee et al. 2014; Takache et al. 2010).

\subsection{PBR Efficiencies and Intensification Principles}

Among the various criteria characterizing the performance of microalgal cultivation systems, biomass productivities per illuminated surface area and per unit volume of culture as well as light to biomass energy conversion efficiency are of primary relevance.

Surface biomass productivity $P_{\mathrm{s}}$ (in $\mathrm{g} \mathrm{m}^{-2} \mathrm{~d}^{-1}$ ) gives the area required to achieve a given amount of biomass produced per day. In light-limited regime, $P_{\mathrm{s}}$ depends only on the PFD received by the culture and on the photosynthetic conversion yield. Productivity is then a function of location, meteorological conditions, and of the system's ability to capture sunlight depending on its design, inclination, and orientation. A useful and simple engineering equation taking into account those parameters was proposed to estimate the maximum surface productivity $P_{\mathrm{s} \text {, max }}$ of any PBR (ponds, flat plate, tubular, ...) knowing only mean yearly solar information (Pruvost and Cornet 2012):

$$
\begin{aligned}
P_{\mathrm{s}, \max }= & \left(1-f_{d}\right) \rho_{\mathrm{M}} M_{X} \bar{\phi}_{X}^{\prime} \frac{2 \alpha}{1+\alpha}\left[\frac{\bar{x}_{d} K}{2} \ln \left[1+\frac{2 \bar{q}}{K}\right]\right. \\
& \left.+\left(1-\bar{x}_{d}\right) \overline{\cos \theta} K \ln \left[1+\frac{\bar{q}}{K \overline{\cos \theta}}\right]\right]
\end{aligned}
$$

where the parameters specific to a given microorganism species are (i) the linear scattering modulus $\alpha$ (default value 0.9 ), (ii) the molar mass $M_{X}$, typically around $0.024 \mathrm{~kg}_{X} / \mathrm{mol}_{X}$, (iii) the half saturation constant for photosynthesis $K$, usually about $100 \mu \mathrm{mol}_{\mathrm{hv}} \mathrm{m}^{-2} \mathrm{~s}^{-1}$, and (iv) $\rho_{\mathrm{M}}$ the maximum energetic yield for photon conversion $(\sim 0.8)$. The location, time of the year, and the ability of the cultivation system to collect light are accounted for by (a) $\bar{x}_{d}$ the fraction of diffuse radiation in 
the total incident solar flux density (PAR), typically around $0.1-0.5$ (b) the cosines of the incident angle $\theta$ onto PBR surface $\overline{\cos \theta}$ usually ranged between 0.4 and 0.7 and (c) the incident PFD $\bar{q}$ (all values here averaged on a given operating period). Here, $f_{\mathrm{d}}$ is the volume fraction of the PBR in the dark which cannot be lit by the incident or scattered PFD because of the system design, as for example when using a recirculating tank.

Equation (2) was originally developed and validated for cyanobacteria (Cornet 2010). It has also been validated for microalgae (Takache et al. 2010). It should be treated as a reliable and convenient tool for calculating the productivities of cultivation systems during design and operation of PBRs. Please note that the logarithmic relation in Eq. (2) accounts for the decrease in photosynthetic conversion efficiency with increasing PFD. Increasing PFD increases the surface productivity of the system but reduced its energy efficiency.

Volumetric biomass productivity $P_{\mathrm{v}}$ (i.e., $\mathrm{g} \mathrm{m}^{-3} \mathrm{~d}^{-1}$ ) gives the volume requested for a given production rate. Its maximum value $P_{\mathrm{V}, \max }$ can be estimated from Eq. (2) according to

$$
P_{\mathrm{V}, \max }=\frac{P_{\mathrm{s}, \max } S_{\text {light }}}{V_{R}}=P_{\mathrm{s}, \text { max }} a_{\text {light }}
$$

This expression introduces an important parameter, $a_{\text {light }}=S_{\text {light }} / V_{\mathrm{R}}$, corresponding to the specific illuminated surface to PBR volume ratio. In contrast to surface productivity which depends only on the ability of the system to collect light, volumetric productivity depends on the engineering design of the cultivation system represented by $a_{\text {light }}$. This parameter can cover two orders of magnitude in practice, ranging from 1 to $10 \mathrm{~m}^{-1}$ for systems presenting culture depths larger than $10 \mathrm{~cm}$ to values of $100 \mathrm{~m}^{-1}$ or larger for systems with thin culture less than $10 \mathrm{~mm}$.

Equations (2) and (3) reveal that the volumetric productivity $P_{\mathrm{v}}$ increases with increasing $a_{\text {light }}$ for constant surface productivity $P_{\mathrm{s}}$ which is independent of $a_{\text {light }}$. Increasing the incident PFD results in an increase in both surface and volumetric productivities providing that adequate light attenuation conditions prevail, as explained in Sect. 2.4. A typical example is given in Fig. 3 for realistic values of $a_{\text {light }}$ corresponding to depths of culture commonly encountered in actual systems and in intensified technologies presenting ultrathin culture (see further description of AlgoFilm(C) technology).

Both surface and volumetric productivities represent the kinetics efficiencies of a cultivation system. Energy efficiency is also a relevant performance indicator for energy applications of PBRs. The rigorous thermodynamic efficiency (the exergetic yield of the PBR) is defined (Cornet et al. 1994) as the ratio of the volumetric chemical power produced over the volumetric light absorption rate $\langle A\rangle$ [Eq. (1)] and can be roughly assimilated to the photosynthetic conversion efficiency of the PBR (PCE). Whereas the kinetics performances increase with increasing PFD, the PCE decreases with PFD because photosynthesis rapidly saturates with light (Cornet 2010; Wilhelm and Selmar 2011). This consideration is the basis of the light dilution principle applied to the design of solar cultivation technologies aiming to 


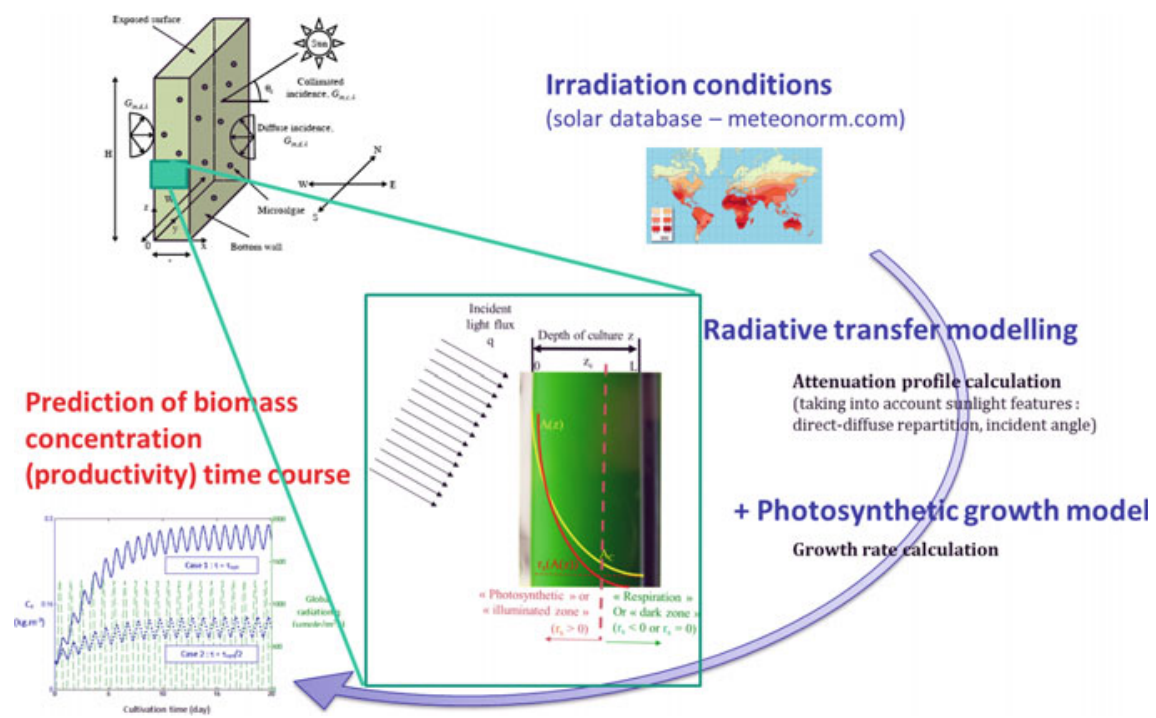

Fig. 3 Overview of the general modeling approach used to simulate solar PBR (for details, see Pruvost and Cornet 2012; Pruvost et al. 2011a, b, 2012)

optimize the rate of biomass produced per unit footprint of PBR. In this case, the surface area used for collecting the sunlight is smaller than the surface area illuminating volumetrically the culture inside the PBR. Their ratio is the so-called geometric dilution ratio which is less than unity. In other words, the PFD inside the culture is lower than the PFD of the captured sunlight. This concept leads then to larger PCE and larger biomass production rates per unit surface area used for collecting light.

\section{The Specific Case of Solar Photobioreactor Engineering}

\subsection{The Use of Sunlight}

Outdoor conditions and the use of sunlight as the primary energy source result in several challenges in the engineering design and control of outdoor cultivation systems. The amount of direct and diffuse solar incident irradiances as well as the strongly time-dependent incident PFD and the associated incident angle have been found to significantly affect the process efficiencies (Pruvost et al. 2011a, 2012). Consequently, although the luminostat regime is the ideal case leading to maximum biomass productivity, it cannot be maintained under solar conditions because of the rapid variations in light with time compared with the biomass concentration (Hindersin et al. 2013; Pruvost et al. 2011a, 2012). Thus, a compromise in design and operation has to be found. This can be achieved, in continuous PBRs, by 
defining for example, a residence time that maximizes the yearly biomass productivity by controlling the temporal evolution of the biomass concentration and the light attenuation in the PBR. Moreover, oversaturating light can be received by cells, especially for high PFD typically larger than $400 \mu$ mole $\mathrm{m}^{-2} \mathrm{~s}^{-1}$. Such PFD are commonly encountered in most Earth's location in the summer. It is known to impair culture health and possibly induce biological drift (i.e., photoinhibition) which can lead to process instability. Strong light attenuation in the PBR is known to have a positive effect as it decreases the amount of light energy received per cell along the depth of the PBR (Carvalho et al. 2011; Hindersin et al. 2013; Torzillo et al. 1996). Overall, these examples reveal that controlling the biomass concentration is a key aspect of optimizing the operation of solar PBR as it directly affects the PBR productivity and its stability.

\subsection{Thermal Regulation Issues}

Like in any biological process, temperature directly influences photosynthesis and microorganism growth. Particularly under solar illumination, closed PBRs tend to overheat and open systems may suffer from evaporation of water under strong incident irradiance. This can be attributed to culture confinement and to the strongly exoenergetic photosynthetic growth (Carvalho et al. 2011; Hindersin et al. 2013; Torzillo et al. 1996; Wilhelm and Selmar 2011). In fact, the thermodynamic efficiency over the PAR region of systems working with low light typical of artificial illumination (100-300 $\mu$ mole $_{\mathrm{h} v} \mathrm{~m}^{-2} \mathrm{~s}^{-1}$ ) is in general below $5 \%$ (Cornet 2010) and decreases to $2 \%$ under large solar irradiance $\left(>500 \mu\right.$ mole $\left._{\mathrm{h} v} \mathrm{~g}^{-2} \mathrm{~s}^{-1}\right)$. As a result, around $95 \%$ of the captured light is converted into heat. In fact, under outdoor conditions, around $50 \%$ of the energy in the solar radiation is contained in the nearand mid-infrared above $750 \mathrm{~nm}$ and directly participates in heating up the culture (Goetz et al. 2011; Hindersin 2013; Hindersin et al. 2013, 2014).

Thermal regulation of PBRs has been widely investigated as a major issue of solar microalgal cultivation (Borowitzka 1999; Grobbelaar 2008; Hindersin et al. 2013, 2014). Unfortunately, without proper thermoregulation, temperatures lethal to living microorganisms can easily be reached inside the PBR. For temperate climates during winter, excessively low temperatures can result in loss of biomass growth and productivity. Then, heating the culture can be beneficial (Hindersin 2013). The appropriate temperature window depends strongly on the species cultivated. However, it typically ranges between 10 and $30{ }^{\circ} \mathrm{C}$.

Various solutions for heating or cooling PBRs are available, depending of the PBR technology, size, and location. Water cooling and/or heating by spraying on the PBR outside surfaces or by direct PBR immersion in a pool are often used (Borowitzka 1999). In temperate regions, cultivation systems can also be placed in greenhouses. Although efficient, those methods can increase the construction and operating costs and negatively impact the environmental footprint through excessive energy and water consumptions. 
Although technical solutions currently exist, PBR temperature control remains a challenge under solar conditions, especially if cost-effective solutions with low energy consumption and year-round operation are sought. This can lead to the need for both cooling and heating. The engineering of the cultivation system is also relevant. For example, Goetz et al. (2011) experimentally and theoretically investigated the effect of various designs of a flat panel PBR. Depending on the configuration, the authors observed a decrease of up to one order of magnitude in the PBR energy consumption. IR filtering, for example, was found to be especially effective at reducing culture overheating. More recently, research efforts have investigated the integration of PBR technology into building façades. This integration offers various benefits regarding thermal management of both PBRs and buildings. Energy exchanges between the building and the PBRs can indeed be designed so as to cool or warm each one of the subsystems. For example, PBRs can filter sunlight in summer to reduce the thermal load on the building. In winter, excess thermal energy in the cultivation system can be used to warm the building. Finally, the added thermal mass of the building can be used to facilitate PBR thermal regulation regardless of the season.

Overall, thermal regulation of PBR depends on the location, the time of year, and on the strain cultivated. Cooling and/or heating requirements have to be estimated (usually in the range $50-200 \mathrm{~W} \mathrm{~m}^{-2}$ ) and the associated thermal solutions should be defined and integrated at the early stage of the system's design. For climate with large variations in outdoor temperature and solar irradiation during the course of a year, it could be beneficial to cultivate different species with optimal growth at different temperatures (Hindersin 2013). This could lead to a significant decrease in energy needs.

\subsection{Modeling for Solar PBR Optimization}

Biophysical modeling of photobiological processes in solar PBRs aims to relate the various design and operating parameters to the productivity and efficiency of microalgal cultivation systems. Such modeling should account for the complex phenomena involved in the process and particularly the coupling between light transfer in the culture and photosynthetic growth. Several recent studies have modeled solar PBR operation with the aim to optimize productivities as a function of PBR design, location, and/or cultivated species (Pruvost et al. 2011a, 2012; Quinn et al. 2011; Slegers et al. 2011, 2013a, b).

Based on in-depth modeling efforts, engineering rules and formulae have been derived to design, optimize, and control PBRs in a predictive and rational way. Those tools are today available for both artificial light sources and sunlight and for systems based on either surface or volumetrically illuminated PBRs. The interested reader is referred to the manuscript by Pruvost et al. (2011a) for a complete description of solar PBR model and to those by Pruvost and coworkers (Pruvost et al. 2011b, 2012; Pruvost and Cornet 2012) for more detailed investigations. 


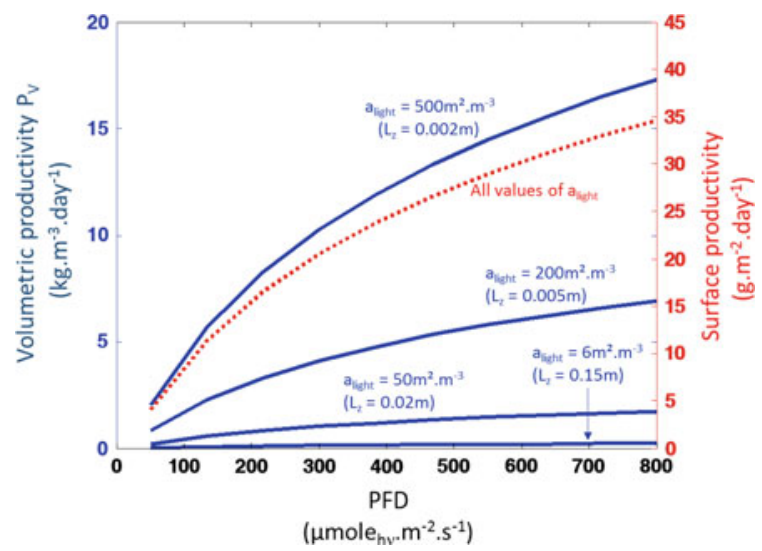

Fig. 4 Maximal volumetric and surface biomass productivities of the microalga Chlamydomonas reinhardtii as a function of incident PFD. Results are given for various typical values of illuminated surface-to-volume ratio $\left(a_{\text {light }}\right)$. Corresponding depths of culture for flat panels are also given for information $\left(a_{\text {light }}=1 / L_{\mathrm{z}}\right)$

In general, current models mainly aim at relating sunlight conditions obtained from meteorological database to growth kinetics so as to predict PBR performances, as illustrated in Fig. 4 (Pruvost et al. 2011a; Quinn et al. 2011; Slegers et al. 2011). These models can provide valuable predictions of productivity for PBR operated during an entire year. They can also assess the influence of various parameters such as PBR location, harvesting strategy, strains cultivated, and the effects of night and day cycles. However, they may be regarded as oversimplified considering the complexity and numerous parameters affecting PBR operation and productivity in outdoor conditions. There is clearly a need to pursue the efforts of developing a set of robust tools for solar cultivation optimization to achieve better accuracy and to extend their applicability to other challenges related to solar PBRs. For example, Slegers et al. (2013a) integrated, in its model of the process, a thermal model able to predict the temporal evolution of the culture temperature under solar conditions and assess its influence on growth. Temperature was found to strongly influence the growth rate. Simulations predicted the range of temperature for a given location as a function of culture volume and thermal inertia of the system. The effect of nonoptimal thermal regulation on the productivity was simulated.

\subsubsection{Model-Based Design of Intensified PBR Technologies}

Modeling can be used to simulate various configurations of PBR for the optimization of their design. This section presents two examples of solar technologies whose development was based on the general methodology described in the previous section. Those technologies, namely AlgoFilm( $\mathrm{C}$ and DiCoFluV(C), are also illustrated in Fig. 1. 
First, the AlgoFilm $\odot$ PBR is based on surface illumination principle. It aims to achieve a very high volumetric productivity and the maximum surface productivity achievable under direct illumination. According to Eq. (3) and Fig. 3, large volumetric productivity can be achieved if the system presents a very large illuminated surface to volume ratio $a_{\text {light }}$. Thus, AlgoFilm $\odot$ PBR is based on a falling film concept with value of $a_{\text {light }}$ of $470 \mathrm{~m}^{2} \mathrm{~m}^{-3}$, corresponding to $2.1 \mathrm{~L}$ per m $\mathrm{m}^{2}$ of illuminated surface area. To fulfill the light limitation condition and to guarantee maximum biomass productivities of the system, various optimization strategies have been explored including (i) PBR hydrodynamics, (ii) gas-liquid mass transfer optimization $\left(\mathrm{CO}_{2}\right.$ dissolution, oxygen removal), (iii) development of thermal regulation devices, and (iv) material selection to prevent biofilm formation. A volumetric productivity of $5.7 \mathrm{~kg} \mathrm{~m}^{-3}$ day $^{-1}$ was experimentally achieved under solar illumination condition with a daily averaged PFD $\bar{q}=270 \mu$ mole $_{\mathrm{hv}} \mathrm{m}^{-2} \mathrm{~s}^{-1}$ typical of the average irradiation conditions in France. Note that the maximum productivity predicted by the model was $5.5 \mathrm{~kg} \mathrm{~m}^{-3}$ day ${ }^{-1}$ (Le Borgne 2011). This confirmed the relevance of the modeling used during the design phase of the cultivation system.

Moreover, the DiCoFluVC concept (Cornet 2010) is based on volumetric illumination with optimized light dilution principle. First, the conception of the optimal layout for the optical fibers with lateral diffusion of light used inside the culture volume was obtained using the constructal approach (Bejan 2000; Bejan and Lorente 2012) and imposing a low PFD to achieve high thermodynamic efficiency (namely 15-18\% in the PAR). This required models of light transfer for simple one-dimensional (Cornet 2010) or complex three-dimensional PBR geometries (Dauchet et al. 2013; Lee et al. 2014). Second, the optimum solar capture area was determined using kinetic model coupling the local light absorption rate $A$ with biomass growth rates based on yearly solar databases. The modeling effort led to a design with 25 Fresnel lenses for a total volume of $30 \mathrm{~L}$ (Fig. 1). The optimal light dilution factor of the incident PFD was found to be relatively constant for any location on earth. But clearly, this concept is more interesting for locations with strong direct illumination. Relatively large volumetric biomass productivities are possible because of the large illuminated surface $a_{\text {light }}$ of roughly $350 \mathrm{~m}^{2} \mathrm{~m}^{-3}$ compensating for the low incident diluted PFD. Nevertheless, this PBR is mainly conceived as an optimal surface biomass productivity concept capable of increasing the surface productivity by an order of magnitude (by unit footprint) in solar conditions compared to conventional direct illumination systems. This corresponds to the maximum thermodynamic efficiency of photosynthesis. Actual performance of the system depends on the optical efficiency of the capture/concentration/ filtration/distribution of light inside the culture vessel. Nowadays, efficiencies between 5 and up to $30 \%$ for the DiCoFluV@ PBR have been reported, reducing the theoretical maximal surface productivities down to around $100 t_{\mathrm{x}} \mathrm{ha}^{-2}$ year $^{-1}$ at the Earth's equator (Cornet 2010). 


\subsubsection{Prediction of PBR Operating Conditions and Optimization of Biomass Productivity}

Modeling can be used to simulate PBR operation under outdoor solar irradiation. Because of variations in the incident illumination, the PBR never reaches a steady state and biomass concentration continuously evolves with time. Figure 4 shows an overview of the general modeling approach used to simulate solar PBRs. By predicting the temporal evolution of the biomass concentration in response to irradiation conditions, modeling of solar PBR operation is especially useful as biomass concentration directly affects light attenuation conditions and the resulting biomass productivity (Hindersin 2013; Hindersin et al. 2014; Slegers et al. 2011; Pruvost et al. 2011a). The effects of the season and of day-night cycles on the process dynamics and biomass productivity can be accounted for. Various engineering parameters can also be easily simulated to identify their optimum values. This includes parameters related to PBR design such as PBR geometry, orientation and inclination, and operating parameters like the residence time applied onto the cultivation system for continuous or semicontinuous cultures (Grognard et al. 2014; Hindersin et al. 2014; Pruvost et al. 2011a, 2012).

Figure 5 shows typical model predictions of the daily surface biomass productivity $P_{\mathrm{S}}$ as a function of the residence time for a continuous flat panel PBR located in Nantes, France for two commonly used microorganisms, namely Chlamydomonas reinhardtii (microalgae, eukaryotic microorganism) and Arthrospira platensis (cyanobacteria, prokaryotic microorganism). Those simulations were conducted for

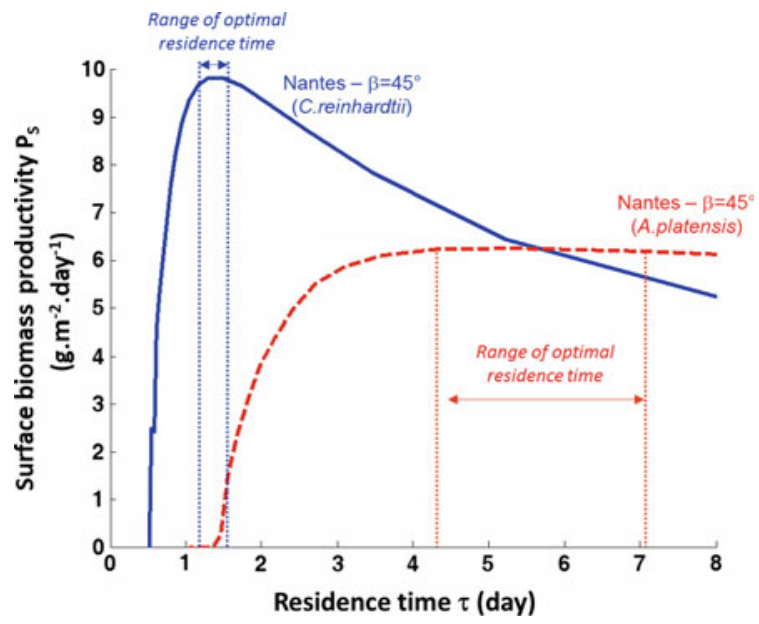

Fig. 5 Yearly average areal productivity of an inclined flat panel PBR $\left(45^{\circ}\right)$ as a function of the residence time applied on the cultivation system operated in continuous mode (Nantes locations, France). Values are given for the microalga $C$. reinhardtii and for the cyanobacteria A. platensis, illustrating the narrower range of residence time to maximize productivity for eukaryotic cells as explained by their sensitivity to dark volumes induced by too high values of residence time values 
light-limited conditions with otherwise optimal growth conditions. An entire year of operation was simulated. Results for $C$. reinhardtii indicate that the maximum daily surface productivity could be achieved over a very narrow range of residence times. By contrast, the daily surface productivity for A. platensis reached a maximum for a large range of residence times. This was due to the fact that A. platensis is less sensitive to the presence of dark regions of the PBR which promote respiration activity (Gonzalez de la Vara and Gomez-Lojero 1986). On the other hand, dark volumes have a strong and negative influence on $C$. reinhardtii growth. Figure 5 also indicates that for either microorganisms, the productivity decreased sharply as the residence time decreased leading to culture washout.

Maximum biomass productivity can be easily achieved in continuous PBR exposed to artificial constant illumination by setting the biomass concentration corresponding to optimal light attenuation conditions (Takache et al. 2010). Under sunlight, the biomass growth rate is not sufficient to compensate for the rapid changes in sunlight intensity. Consequently, light attenuation conditions are never optimal. The optimal value of the residence time illustrated in Fig. 5 represents the best compromise to achieve maximum biomass productivity over the year of operation.

Because of variations in the incident irradiation, a wide range of light attenuation conditions can be encountered inside the culture volume during the course of a day. This can affect the process stability. For example, promoting small residence time to reduce the extent of dark zone favors low biomass concentration. This also reduces light attenuation and possibly impairs the process stability for periods where oversaturating light is encountered, such as at noon in the summer. A practical advice consists of promoting light attenuation by relying on large biomass concentration. For example, Hindersin et al. (2013) recommended a minimum value of biomass concentration for a given PFD incident on a solar PBR with sun-tracking capabilities, to maintain sufficient light attenuation. However, this approach results in a decrease in biomass productivity particularly for species with large respiration activity under illumination, as previously discussed. Again, a compromise has to be found, between the process productivity and its stability and robustness.

A first attempt to set rational strategies to achieve this compromise can be proposed. Light transmission through the PBR can be considered as an indicator of insufficient PFD attenuation, as light is not fully absorbed by the culture. Based on simulations of the process operation, the number of hours when light transmission through a continuous PBR occurs during a year of operation is calculated. Figure 6 shows the fraction of the time when light transmission is nonzero as a function of the residence time imposed to the cultivation system simulated in Fig. 5. It indicates that the number of hours when light transmission occurs is strongly influenced by the residence time because of its direct dependence on biomass concentration. For example, long residence time results in high biomass concentration and strong PFD attenuation. However, the evolutions of biomass productivity and light transmission with the residence time are different. For example, at optimal residence time for maximal biomass productivity of $C$. reinhardtii, the light transmission regime prevails $50 \%$ of the time when the cultivation system is illuminated. This relatively 


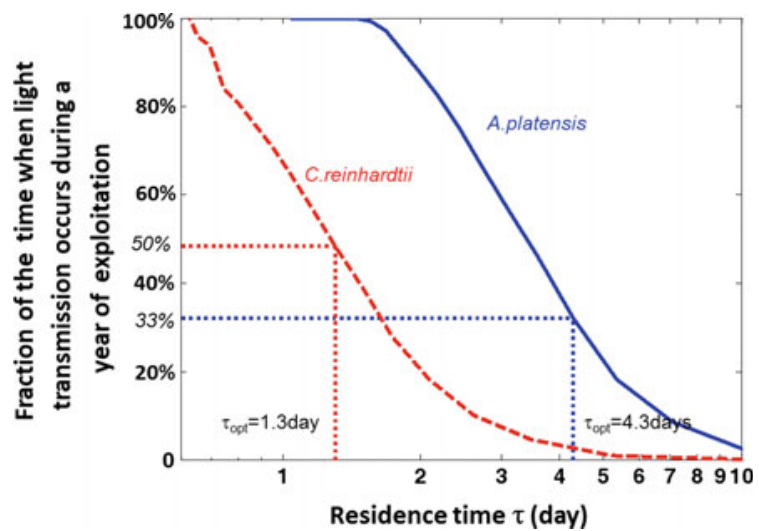

Fig. 6 Distribution of the number of hours per year when light transmission occurs, as a function of the residence time applied in the cultivation system (Nantes location, inclined PBR at $45^{\circ}, C$. reinhardtii). Values are normalized with respect to the total number of lightened hours in the year, i.e., $4355 \mathrm{~h}$ for Nantes location

large fraction would certainly lead to a significant risk of photoinhibition and culture drift, and potentially process instability and loss of efficiency. This risk can be reduced by increasing the residence time to obtain larger biomass concentration. For example, if the residence time is doubled, the PBR presents non-zero light transmission during only $15 \%$ of illuminated hours, which can be considered acceptable as a first-order approximation. This corresponds to a limited loss of productivity of about $10-15 \%$. These results illustrate the need to find a compromise between maximizing biomass productivity and maintaining stable production conditions by setting the appropriate residence time and the associate biomass concentration in the cultivation system. Then, modeling seems especially useful since it can predict the evolution of the parameters defining the state of the culture so as to determine the optimum operating conditions leading to both a robust and efficient production.

\subsubsection{Definition of Optimal Concentration to Maximize PBR Productivity}

Biophysical models previously mentioned can be used to derive simple and practical analytical expressions and rules of thumb to determine the maximum surface and volumetric biomass productivities achievable by the PBR, thanks to Eqs. (2) and (3), respectively. Other models have also been developed to find the optimum PBR dimensions and/or microorganism concentration for a given species. The last two parameters have typically been treated and optimized separately (Pruvost et al. 2011a; Quinn et al. 2011; Slegers et al. 2011). However, based on the observation that PBR productivities were strongly influenced by light attenuation conditions, 
Lee et al. (2014) used the concept of absorption optical thickness $\tau_{\lambda}$ defined as the product $\tau_{\lambda}=\mathrm{Ea}_{\lambda} C_{X} / a_{\text {light }}$ (dimensionless). Here, $\mathrm{Ea}_{\lambda}$ is the specific absorption coefficient for a given species under the growth conditions considered, $C_{X}=C_{X}(t=0)$ is the initial biomass concentration, and $a_{\text {light }}=S_{\text {light }} / V_{\mathrm{R}}$ is the illuminated surface area per unit volume of PBR, also used in Eq. (3). Figure 7a shows the surface productivity $P_{s}$ of horizontal PBR, growing $C$. reinhardtii and operated in batch mode during a summer day in Los Angeles, CA as a function of initial biomass concentration for different values of $a_{\text {light }}=1 / L$ where $L$ is the depth

Fig. 7 Surface productivity for horizontal PBR presenting different values of depth $L$ and of $a_{\text {light }}=1 / L$ as a function of (a) initial biomass concentration $C_{X}$ and (b) for the ratio $C_{X} / a_{\text {light }}$
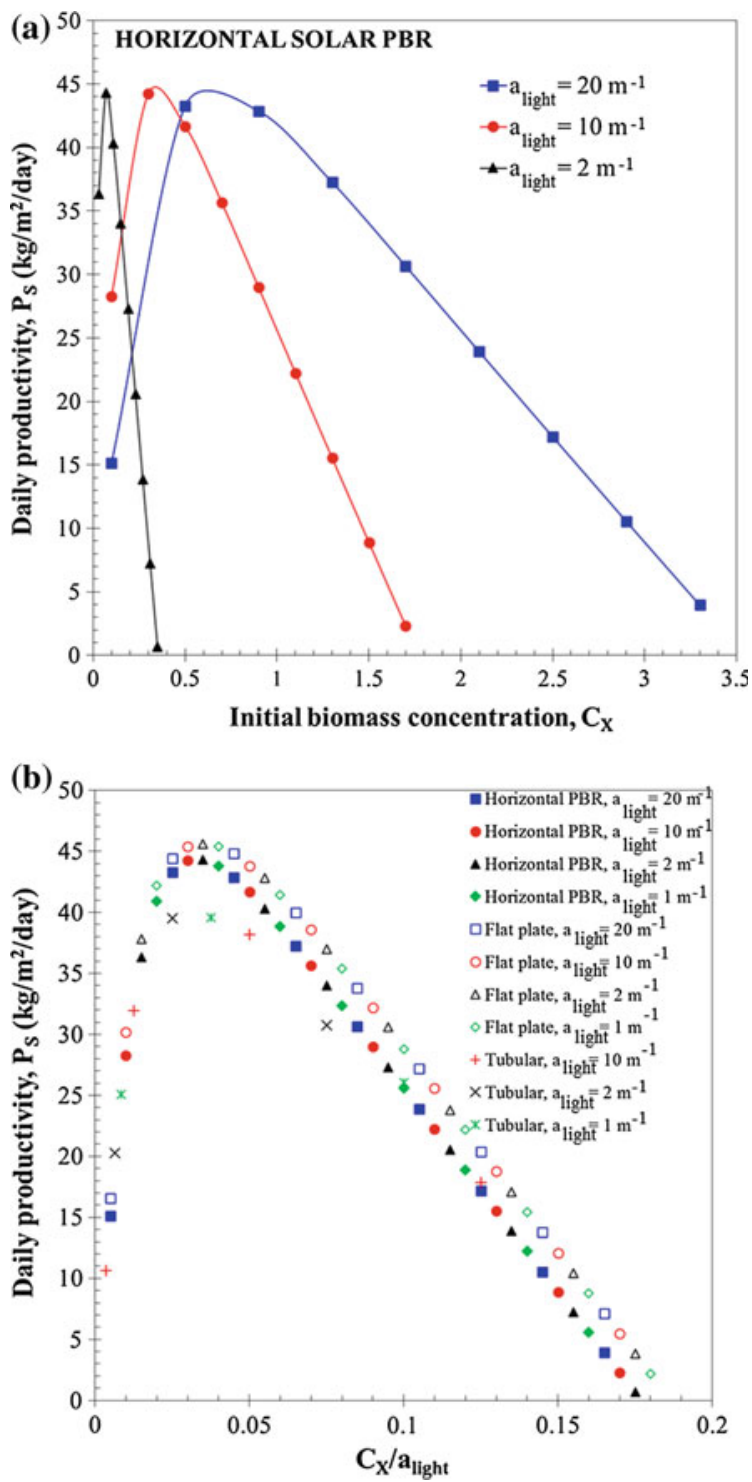
of the pond. It indicates that for a given depth, there exists an optimal initial concentration for which $P_{S}$ is maximal. Figure $7 \mathrm{~b}$ shows the same data but plotted as a function of $C_{X} / a_{\text {light }}$ as well as those for flat plate and tubular PBRs. It is evident that the daily surface productivity $P_{s}$ was a unique function of the ratio $C_{X} /$ $a_{\text {light }}$. What is more, the function $P_{s}\left(C_{X} / a_{\text {light }}\right)$ for $C$. reinhardtii was the same for outdoor horizontal PBR (open ponds), vertical flat plate, and tubular PBRs operated in batch mode. In addition, the validity of this approach was also established for experimental data (Pruvost et al. 2011b) and other simulation results based on different models (Pruvost et al. 2011a; Quinn et al. 2011; Slegers et al. 2011) for different microorganisms and PBRs operated in continuous mode. The PBR absorption optical thickness, represented by $C_{X} / a_{\text {light }}$ for a given species, constitutes a convenient parameter for designing (via $\left.a_{\text {light }}=S_{\text {light }} / V_{R}\right)$ and operating $\left(\right.$ via $\left.C_{X}\right)$ these PBRs to achieve their maximum productivity $P_{s, \max }$ predicted by Eq. (2).

\section{Conclusions}

This chapter has reviewed the various parameters which one should consider in designing and operating large microorganism cultivation systems. Open systems constitute a simple and mature technology already deployed at industrial scale. By contrast, closed PBRs can be regarded as more complex systems, mainly due to the influence of light on the process. However, the main challenges have been identified and some robust engineering solutions have been recently proposed. Their use in the design and control of solar PBRs was illustrated in this chapter. Research efforts have to be pursued to develop solar PBR technologies in order to achieve their maximum theoretical performance. This is a prerequisite to compensate for the higher cost associated with the confinement of the culture to prevent contamination. These efforts should focus on the closely connected areas of biophysical modeling, engineering design, and operation and control.

Acknowledgments This work was supported by the French National Research Agency project DIESALG (ANR-12-BIME-0001-02) for biodiesel production based on solar production of microalgae, and is part of the French "BIOSOLIS" research program on developing photobioreactor technologies for mass-scale solar production (http://www.biosolis.org/). Laurent Pilon is grateful to the Région Pays de la Loire for the Research Chair for International Junior Scientists.

\section{References}

Acién Fernández, F. G., Fernández Sevilla, J. M., Sánchez Pérez, J. A., Molina Grima, E. \& Chisti, Y. (2001). Airlift-driven external-loop tubular photobioreactors for outdoor production of microalgae: Assessment of design and performance. Chemical Engineering Science, 56, 27212732.

Aiba, S. (1982). Growth kinetics of photosynthetic microorganisms. Advances in Biochemical Engineering and Biotechnology, 23, 85-156. 
Bejan, A. (2000). Shape and structure, from engineering to nature. Cambridge, UK: Cambridge University Press.

Bejan, A., \& Lorente, S. (2012). The physics of spreading ideas. International Journal of Heat and Mass Transfer, 55, 802-807.

Borowitzka, M. A. (1999). Commercial production of microalgae: ponds, tanks, and fermenters. Progress in Industrial Microbiology, 35, 313-321.

Carvalho, A. P., Meireles, L. A., \& Malcata, F. X. (2006). Microalgal reactors: A review of enclosed system designs and performances. Biotechnology Progress, 22, 1490-1506.

Carvalho, A. P., Silva, S. O., Baptista, J. M., \& Malcata, F. X. (2011). Light requirements in microalgal photobioreactors: An overview of biophotonic aspects. Applied Microbiology and Biotechnology, 89, 88-1275.

Cassano, A. E., Martin, C. A., Brandi, R. J., \& Alfano, O. M. (1995). Photoreactor analysis and design: fundamentals and applications. Industrial and Engineering Chemistry Research, 34, 2155-2201.

Chini Zitelli, G. C., Pastorelli, R., \& Tredici, M. R. (2000). A modular flat panel photobioreactor (MFPP) for indoor mass cultivation of Nannochloropsis sp. under artificial illumination. Journal of Applied Phycology, 12, 521-526.

Chini Zittelli, G., Rodolfi, L., Biondi, N., \& Tredici, M. R. (2006). Productivity and photosynthetic efficiency of outdoor cultures of Tetraselmis suecica in annular columns. Aquaculture, 261, 932-943.

Cornet, J. F., Dussap, C. G., Cluzel, P., \& Dubertret, G. (1992a). A structured model for simulation of cultures of the cyanobacterium Spirulina platensis in photobioreactors. 1. Coupling between light transfer and growth kinetics. Biotechnology and Bioengineering, 40, 817-825.

Cornet, J. F., Dussap, C. G., Cluzel, P., \& Dubertret, G. (1992b). A structured model for simulation of cultures of the cyanobacterium Spirulina platensis in photobioreactors. 2. Identification of kinetic parameters under light and mineral limitations. Biotechnology and Bioengineering, 40, 826-834.

Cornet, J. F., Dussap, C. G., \& Gros, J. B. (1994). Conversion of radiant light energy in photobioreactors. AIChE Journal, 40, 1055-1066.

Cornet, J. F., Dussap, C. G., \& Gros, J. B. (1995). A simplified monodimensional approach for modeling coupling between radiant light transfer and growth kinetics in photobioreactors. Chemical Engineering Science, 50, 1489-1500.

Cornet, J. F., \& Dussap, C. G. (2009). A simple and reliable formula for assessment of maximum volumetric productivities in photobioreactors. Biotechnology Progress, 25, 424-435.

Cornet, J.-F. (2010). Calculation of optimal design and ideal productivities of volumetrically lightened photobioreactors using the constructal approach. Chemical Engineering Science, 65, 985-998.

Csogör, Z., Herrenbauer, M., Schmidt, K., \& Posten, C. (2001). Light distribution in a novel photobioreactor - modelling for optimization. Journal of Applied Phycology, 13, 325-333.

Dauchet, J., Blanco, S., Cornet, J. F., El Hafi, M., Eymet, V., \& Fournier, R. (2013). The practice of recent radiative transfer Monte Carlo advances and its contribution to the field of microorganisms cultivation in photobioreactors. Journal of Quantitative Spectroscopy \& Radiative Transfer, 128, 52-59.

Degrenne, B., Pruvost, J., Christophe, G., Cornet, J. F., Cogne, G., \& Legrand, J. (2010). Investigation of the combined effects of acetate and photobioreactor illuminated fraction in the induction of anoxia for hydrogen production by Chlamydomonas reinhardtii. International Journal of Hydrogen Energy, 35, 10741-10749.

Doucha, J., \& Livansky, K. (2006). Productivity, $\mathrm{CO}_{2} / \mathrm{O}_{2}$ exchange and hydraulics in outdoor open high density microalgal (Chlorella sp.) photobioreactorsoperated in a Middle and Southern European climate. Journal of Applied Phycology, 18, 811-826.

Duffie, J. A., \& Beckman, W. A. (2006). Solar engineering of thermal processes. New York, NY: John Wiley \& Sons.

Goetz, V., LE Borgne, F., Pruvost, J., Plantard, G. \& Legrand, J. 2011. A generic temperature model for solar photobioreactors. Chemical Engineering Journal, 175, 443-449. 
Gonzalez De La Vara, L., \& Gomez-lojero, C. (1986). Participation of plastoquinone, cytochrome c553 and ferredoxin-NADP + oxido reductase in both photosynthesis and respiration in Spirulina maxima. Photosynthesis Research, 8, 65-78.

Molina Grima, E., Acien Fernandez, F. G., Garcia Camacho, F., \& Chisti, Y. (1999). Photobioreactors: light regime, mass transfer, and scaleup. Jounal of Biotechnology, 70, 231-247.

Grobbelaar, J. U. (2008). Factors governing algal growth in photobioreactors: the "open" versus "closed" debate. Journal of Applied Phycology, 21, 489-492.

Grognard, F., Akhmetzhanov, A. R., \& Bernard, O. (2014). Optimal strategies for biomass productivity maximization in a photobioreactor using natural light. Automatica, 50, 359-368.

Hindersin, S. 2013. Photosynthetic efficiency of microalgae and optimization of biomass production in photobioreactors. PhD Thesis, Hamburg University.

Hindersin, S., Leupold, M., Kerner, M., \& Hanelt, D. (2013). Irradiance optimization of outdoor microalgal cultures using solar tracked photobioreactors. Bioprocess and Biosystems Engineering, 36, 55-345.

Hindersin, S., Leupold, M., Kerner, M., \& Hanelt, D. (2014). Key parameters for outdoor biomass production of Scenedesmus obliquus in solar tracked photobioreactors. Journal of Applied Phycology, 26, 2315-2325.

Hsieh, C. H., \& Wu, W. T. (2009). A novel photobioreactor with transparent rectangular chambers for cultivation of microalgae. Biochemical Engineering Journal, 46, 300-305.

Hu, Q., Guterman, H., \& Richmond, A. (1996). A flat inclined modular photobioreactor for outdoor mass cultivation of photoautotrophs. Biotechnology and Bioengineering, 51, 51-60.

Ifrim, G. A., Titica, M., Cogne, G., Boillereaux, L., Legrand, J., \& CARAMAN, S. 2014. Dynamic $\mathrm{pH}$ model for autotrophic growth of microalgae in photobioreactor: A tool for monitoring and control purposes. AIChE Journal, 60, 585-599.

Kandilian, R., Lee, E., \& Pilon, L. (2013). Radiation and optical properties of Nannochloropsis oculata grown under different irradiances and spectra. Bioresource Technology, 137, 63-73.

Kandilian, R., Tsao, T.-C., \& Pilon, L. (2014). Control of incident irradiance on a batch operated flat-plate photobioreactor. Chemical Engineering Science, 119, 99-108.

Le Borgne, F. (2011). Développement d'un photobioréacteur solaire intensifié en vue de la production à grande échelle de biomasse microalgale. Thèse de doctorat: Université de Nantes.

Lee, E., Pruvost, J., He, X., Munipalli, R., \& Pilon, L. (2014). Design tool and guidelines for outdoor photobioreactors. Chemical Engineering Science, 106, 18-29.

Lee, Y.-K., \& Low, C.-S. (1991). Effect of photobioreactor inclination on the biomass productivity of an outdoor algal culture. Biotechnology and Bioengineering, 38, 995-1000.

Molina, E., Fernández, J., Acién, F. G., \& Chisti, Y. (2001). Tubular photobioreactor design for algal cultures. Journal of Biotechnology, 92, 113-131.

Morweiser, M., Kruse, O., Hankamer, B., \& Posten, C. (2010). Developments and perspectives of photobioreactors for biofuel production. Applied Microbiology and Biotechnology, 87, 301-1291.

Ogbonna, J. C., Yada, H., Masui, H., \& Tanaka, H. (1996). A novel internally illuminated stirred tank photobioreactor for large-scale cultivation of photosynthetic cells. Journal of Fermentation and Bioengineering, 82, 61-67.

Oswald, W. J. 1988. Large-scale algal culture systems (engineering aspects). In Borowitska, M. (ed.) Microalgal Biotechnology. Cambridge: Cambridge University Press.

Pilon, L., Berberoglu, H., \& Kandilian, R. (2011). Radiation transfer in photobiological carbon dioxide fixation and fuel production by microalgae. Journal of Quantitative Spectroscopy \& Radiative Transfer, 112, 2639-2660.

Pruvost, J., Van Vooren, G., Cogne, G., \& Legrand, J. (2009). Investigation of biomass and lipids production with Neochloris oleoabundans in photobioreactor. Bioresource Technology, 100, 5988-5995.

Pruvost, J. 2011. Cultivation of algae in photobioreactors for biodiesel production. In: USA, E. I. (ed.) Biofuels: alternative feedstocks and conversion processes. A Pandey, C Larroche, SC Ricke and CG Dussap. 
Pruvost, J., Cornet, J. F., Goetz, V., \& Legrand, J. (2011a). Modeling dynamic functioning of rectangular photobioreactors in solar conditions. AIChE Journal, 57, 1947-1960.

Pruvost, J., Van Vooren, G., Le Gouic, B., Couzinet-Mossion, A., \& Legrand, J. (2011b). Systematic investigation of biomass and lipid productivity by microalgae in photobioreactors for biodiesel application. Bioresource Technology, 102, 150-158.

Pruvost, J. \& Cornet, J. F. 2012. Knowledge models for engineering and optimization of photobioreactors. In: C.Walter, C. P. A. (ed.) Microalgal Biotechnology. De Gruyter GmbH \& Co. KG.

Pruvost, J., Cornet, J. F., Goetz, V., \& Legrand, J. (2012). Theoretical investigation of biomass productivities achievable in solar rectangular photobioreactors for the cyanobacterium Arthrospira platensis. Biotechnology Progress, 28, 699-714.

Pulz, O. (2001). Photobioreactors: production systems for phototrophic microorganisms. Applied Microbiology and Biotechnology, 57, 287-293.

Quinn, J., De Winter, L., \& Bradley, T. (2011). Microalgae bulk growth model with application to industrial scale systems. Bioresource Technology, 102, 5083-5092.

Richmond, A., \& Cheng-Wu, Z. (2001). Optimization of a flat plate glass reactor for mass production of Nannochloropsis sp. outdoors. Journal of Biotechnology, 85, 259-269.

Richmond, A. (2004a). Handbook of microalgal culture: biotechnology and applied phycology. Oxford: UK, Blackwell Sciences Ltd.

Richmond, A. (2004b). Principles for attaining maximal microalgal productivity in photobioreactors: An overview. Hydrobiologia, 512, 33-37.

Slegers, P. M., Wijffels, R. H., Van Straten, G., \& Van Boxtel, A. J. B. (2011). Design scenarios for flat panel photobioreactors. Journal of Applied Energy, 88, 3342-3353.

Slegers, P. M., Lösing, M. B., Wijffels, R. H., Van Straten, G., \& Van Boxtel, A. J. B. (2013a). Scenario evaluation of open pond microalgae production. Algal Research, 2, 358-368.

Slegers, P. M., Van Beveren, P. J. M., Wijffels, R. H., Van Straten, G. \& Van Boxtel, A. J. B. (2013b). Scenario analysis of large scale algae production in tubular photobioreactors. Applied Energy, 105, 395-406.

Takache, H., Christophe, G., Cornet, J. F., \& Pruvost, J. (2010). Experimental and theoretical assessment of maximum productivities for the microalgae Chlamydomonas reinhardtii in two different geometries of photobioreactors. Biotechnology Progress, 26, 431-440.

Torzillo, G., Accolla, P., Pinzani, E., \& Masojidek, J. (1996). In situ monitoring of chlorophyll fluorescence to assess the synergistic effect of low temperature and high irradiance stresses in Spirulina cultures grown outdoors in photobioreactors. Journal of Applied Phycology, 8, 283-291.

Ugwu, C. U., Aoyagia, H., \& Uchiyamaa, H. (2008). Photobioreactors for mass cultivation of algae. Bioresource Technology, 99, 4021-4028.

Wilhelm, C., \& Selmar, D. (2011). Energy dissipation is an essential mechanism to sustain the viability of plants: The physiological limits of improved photosynthesis. Journal of Plant Physiology, 168, 79-87.

Zijffers, J. W., Janssen, M., Tramper, J., \& Wijffels, R. H. (2008). Design process of an area-efficient photobioreactor. Marine Biotechnology, 10, 404-415. 